\title{
Characteristics, Properties and Analytical Methods of Amoxicillin: A Review with Green Approach
}

\section{Bianca Aparecida de Marco, Jéssica Sayuri Hisano Natori, Stefany Fanelli, Eliane Gandolpho Tótoli \& Hérida Regina Nunes Salgado}

To cite this article: Bianca Aparecida de Marco, Jéssica Sayuri Hisano Natori, Stefany Fanelli, Eliane Gandolpho Tótoli \& Hérida Regina Nunes Salgado (2017) Characteristics, Properties and Analytical Methods of Amoxicillin: A Review with Green Approach, Critical Reviews in Analytical Chemistry, 47:3, 267-277, DOI: 10.1080/10408347.2017.1281097

To link to this article: https://doi.org/10.1080/10408347.2017.1281097

Accepted author version posted online: 12

Jan 2017.

Published online: 03 Feb 2017.

Submit your article to this journal 단

Џlll Article views: 336

Citing articles: 13 View citing articles 지 


\title{
Characteristics, Properties and Analytical Methods of Amoxicillin: A Review with Green Approach
}

\author{
Bianca Aparecida de Marco (D), Jéssica Sayuri Hisano Natori, Stefany Fanelli, Eliane Gandolpho Tótoli, \\ and Hérida Regina Nunes Salgado
}

Department of Pharmaceutics, School of Pharmaceutical Sciences, Universidade Estadual Paulista, Araraquara, São Paulo, Brazil

\begin{abstract}
Bacterial infections are the second leading cause of global mortality. Considering this fact, it is extremely important studying the antimicrobial agents. Amoxicillin is an antimicrobial agent that belongs to the class of penicillins; it has bactericidal activity and is widely used in the Brazilian health system. In literature, some analytical methods are found for the identification and quantification of this penicillin, which are essential for its quality control, which ensures maintaining the product characteristics, therapeutic efficacy and patient's safety. Thus, this study presents a brief literature review on amoxicillin and the analytical methods developed for the analysis of this drug in official and scientific papers. The major analytical methods found were high-performance liquid chromatography (HPLC), ultra-performance liquid chromatography (U-HPLC), capillary electrophoresis and iodometry and diffuse reflectance infrared Fourier transform. It is essential to note that most of the developed methods used toxic and hazardous solvents, which makes necessary industries and researchers choose to develop environmental-friendly techniques to provide enhanced benefits to environment and staff.
\end{abstract}

\section{KEYWORDS}

Amoxicillin; analytical methods; antimicrobial; green chemistry; penicillins

\section{Introduction}

Due to the multiplicity of microorganisms, studies related to the development of new antimicrobial agents should be increasingly encouraged, in order to contribute to the treatment of infectious diseases and combating bacterial resistance (Hoefel and Lautert, 2006).

An important type of antibiotic was discovered accidentaly in 1928 by Alexander Fleming. While studying variants of Staphylococcus, Fleming observed a zone of inhibition formed around a fungus that was contaminating his cultures. The antibacterial substance was named penicillin, since it was produced by the Penicillium fungus (Brunton et al., 2012; Williams and Lemke, 2013).

Penicillins can be classified into several subcategories, which are differentiated by the chemical structures and antimicrobial activities (Brazil, 2016a). Amoxicillin, which belongs to the aminopenicillins subcategory, was synthesized in 1964 by the introduction of a $p$-hydroxyl group in the side chain of ampicillin, which is another aminopenicillin. In this way, the oral absorption was improved and the side effects, such as diarrhea, were minimized, compared to ampicillin (Holten and Onsusko, 2000; Rolinson and Geddes, 2007; Kaur et al., 2011).

Amoxicillin belongs to the RENAME list (Brazilian List of Essential Medicines) and is widely prescribed in clinical practice for the treatment of otitis media and sinusitis. It is also indicated for the treatment of tonsil, throat, larynx, pharynx, bronchi, lungs and skin infections (Miller, 2002; Rolinson and Geddes, 2007; Kaur et al., 2011).

This antimicrobial agent is easily inactivated by $\beta$-lactamases; therefore, it can be used in combination with $\beta$-lactamase inhibitors, such as clavulanic acid (Miller, 2002; Brazil, 2016b).

In Brazil, two tradenames for amoxicillin can be found as reference drugs, Amoxil ${ }^{\mathrm{TM}}$ and Amoxil $\mathrm{BD}^{\mathrm{TM}}$. They are commercialized in the pharmaceutical dosage forms of powder for oral suspension, hard gelatin capsule and coated tablet. For the combination of amoxicillin and clavulanic acid, the commercial names are Clavulin ${ }^{\mathrm{TM}}$, Clavulin $\mathrm{BD}^{\mathrm{TM}}$, Clavulin $\mathrm{ES}^{\mathrm{TM}}$ and Clavulin $\mathrm{IV}^{\mathrm{TM}}$, in powder for oral suspension, coated tablet, powder for extemporaneous preparation and lyophilized powder for solution for injection. All these medicines are produced by Glaxosmithkline pharmaceutical company (GSK) (Brasil, 2016c).

In addition to the association with clavulanic acid, amoxicillin is also found associated with sulbactam, as Trifamox $\mathrm{IBL}^{\mathrm{TM}}$ and Trifamox IBL $\mathrm{BR}^{\mathrm{TM}}$, in the pharmaceutical dosage forms of powder for solution for injection, coated tablet and powder for extemporaneous preparation, produced by Bagó do Brasil Laboratories (Brazil, 2016d).

Medley Pharmaceutical Industry produces an association of lansoprazole, clarithromycin and amoxicillin trihydrate, named Pyloripac $^{\mathrm{TM}}$, as well as an association of lansoprazole, levofloxacin and amoxicillin trihydrate, named Pyloripac Retrat ${ }^{\mathrm{TM}}$. 
Both are marketed in the pharmaceutical dosage form of hard gelatin capsule and delayed release coated tablet. The pharmaceutical company Libbs produces an association of omeprazole, clarithromycin and amoxicillin, named Erradic U.G. ${ }^{\text {TM }}$, in the pharmaceutical forms of hard gelatin capsule and coated tablet (Brazil, 2016d).

Due to high demand about the use of amoxicillin worldwide, studies involving its quality control are highly encouraged in order to ensure the standards of reliability and effectiveness of this drug, minimizing the risks for the population. In this context, the development of reliable analytical methods is increasingly recognized and required (La Roca et al., 2007). In addition, antimicrobials with lack of quality contribute to the emergence of the serious problem of bacterial resistance.

Considering the importance of amoxicillin for the clinical practice, this paper aims to review the chemical, pharmaceutical and clinical aspects of this antimicrobial, as well as reviewing the analytical methods described in literature for its analysis. The research was carried out using MEDLINE, Science Direct and Scifinder databases, from January 1950 to June 2016, using English language restriction. The main terms used were: 'amoxicillin' in combination with 'HPLC', 'U-HPLC', 'UPLC', 'spectrometry', 'spectrophotometry', 'agar diffusion', 'analytical methods', 'quantitative analysis', 'microbiological assay', among others. The studies included in this review met the following criteria: original data; data regarding the use of amoxicillin and data regarding analytical methods.

\section{Amoxicillin}

\section{Structural forms}

Amoxicillin is available in three different forms: anhydrous, sodium and trihydrate (Argentina Pharmacopoeia, 2003; BP, 2012; Brazilian Pharmacopoeia, 2010; EP, 2013; IP, 2007; JP, 2011; USP 37, 2014). Figure 1 illustrates the chemical structures.

\section{Mechanism of action}

All $\beta$-lactam antibiotics are bactericidal agents that interfere with the biosynthesis of peptidoglycan, one of the constituents of the bacterial cell wall. This structure is necessary for the maintenance of cell integrity, among other vital processes (Miller, 2002; Kaur et al., 2011). The peptidoglycan is composed of two alternate amino sugars ( $\mathrm{N}$-acetylglucosamine and $\mathrm{N}$-acetylmuramic acid) and a peptide of five amino acids ending in D-Alanyl-D-Alanine, which is attached to the $\mathrm{N}$-acetylmuramic acid. This polymer provides rigid mechanical stability due to its highly crosslinked structure (Petri, 2006).

Amoxicillin binds to the penicillin binding protein $1 \mathrm{~A}$ (PBP-1A), which is located inside the bacterial cell wall, and inactivates the penicillin-sensitive transpeptidase by acylation of its C-terminal domain. This interaction is possible because amoxicillin has a structure similar to the DAlanyl-D-Alanine, the original substrate for this enzyme. This enzyme inhibition occurs in the last step of the synthesis of the bacterial cell wall, thus preventing cross-linking of two linear peptidoglycan chains (Petri, 2006). Structural destabilization of the cell wall leads to the formation of spheroplasts and rapid occurrence of lysis mediated autolytic enzymes (Petri, 2006; Kaur et al., 2011).

\section{Microbiological spectrum and clinical use}

Amoxicillin is active against Streptococcus pyogenes and many strains of Streptococcus pneumoniae and Haemophilus influenzae, which are causative microorganisms of bacterial infections of the upper respiratory tract. For this reason, it is indicated in cases of tonsil, throat, larynx and pharynx infections and may also act on the bronchi and lungs (Petri, 2006; Kaur et al., 2011). Amoxicillin is the most active of all oral $\beta$-lactam antibiotics against S. pneumoniae, and it is first choice drug for the treatment of maxillary sinusitis and otitis media (Miller, 2002).

This penicillin may also be used for the treatment of uncomplicated urinary tract infections caused by enterobacteria such as Escherichia coli. Amoxicillin is also used as prophylaxis against bacterial endocarditis and before genitourinary and gastrointestinal procedures (Miller, 2002; Petri, 2006; Rolinson, Geddes 2007; Kaur et al., 2011).

\section{Pharmacokinetics and pharmacodynamics}

Amoxicillin has high oral bioavailability (70-90\%), which is not interfered by the presence of food in the gastrointestinal tract. The plasma concentration peaks occur after 1-2 hours, depending on the dose. About $20 \%$ of amoxicillin is bound to plasma proteins (Williams and Lemke, 2013).

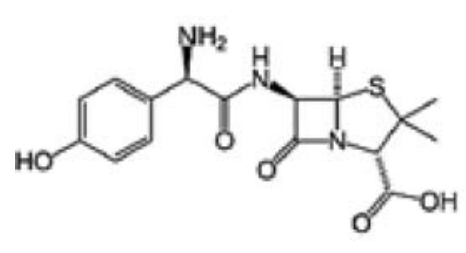

$\mathrm{C}_{16} \mathrm{H}_{19} \mathrm{~N}_{3} \mathrm{O}_{35} \mathrm{~S}$ CAS 26787-78-0

a)

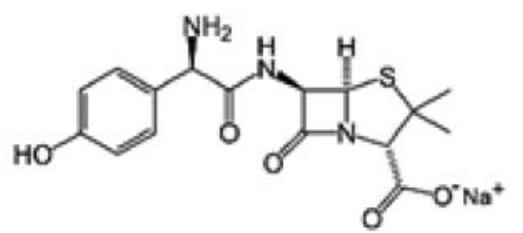

$\mathrm{C}_{16} \mathrm{H}_{19} \mathrm{NaN}_{3} \mathrm{O} s \mathrm{~S}$

CAS 34642-77-8

b)

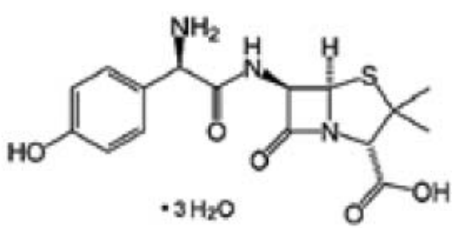

$\mathrm{C}_{16} \mathrm{H}_{19} \mathrm{~N}_{3} \mathrm{O} 5 \mathrm{~S} \cdot 3\left(\mathrm{H}_{2} \mathrm{O}\right)$ CAS 61336-70-7

Figure 1. Chemical structures of amoxicillin anhydrous (a), amoxicillin sodium (b) and amoxicillin trihydrate (c). 
Table 1. Analytical methods described in literature for determination of amoxicillin in pharmaceutical dosage forms and raw material.

\begin{tabular}{|c|c|c|c|c|}
\hline Method & Conditions & Detection system & Matrix & Reference \\
\hline Colorimetry & $\begin{array}{l}\text { The system was based on aggregation of citrate-capped gold } \\
\text { nanoparticles in acetate buffer }(\mathrm{pH}=4.5) \text { in the presence of } \\
\text { the degradation product of amoxicillin (DPAMX). The } \\
\text { absorbance ratio at the wavelength of } 660 \text { and } 525 \mathrm{~nm}\left(\mathrm{~A}_{660} /\right. \\
\mathrm{A}_{525} \text { ) was chosen as the analytical signal indirectly related to } \\
\text { amoxicillin concentration. }\end{array}$ & $\begin{array}{l}\text { UV/Vis }(600- \\
\quad 700 \mathrm{~nm})\end{array}$ & Capsules & Akhond et al. (2015) \\
\hline HPLC & $\begin{array}{l}\text { Thermosil } \mathrm{C}_{18} \text { analytical column }(250 \times 4.6 \mathrm{~mm} ; 5 \mu \mathrm{m}) \text {. Mobile } \\
\text { phase: potassium dihydrogen phosphate buffer (adjusted to } \\
\text { pH } 3 \text { by ortho phosphoric acid) and methanol }(70: 30, \mathrm{v} / \mathrm{v}) \text {. } \\
\text { Flow rate: } 1.0 \mathrm{~mL} / \mathrm{min} \text {. Rt: } 2.582 \mathrm{~min} \text {. }\end{array}$ & UV/DAD (225 nm) & Bulk and capsules & Konari and Jacob (2015) \\
\hline U-HPLC & $\begin{array}{l}\text { Shim-Pack XR-ODS analytical column }(50 \times 2.0 \mathrm{~mm} ; 2.2 \mu \mathrm{m}) \text {. } \\
\text { Mobile phase: methanol and } 0.0025 \mathrm{M} \text { ammonium acetate, in } \\
\text { the initial ratio } 40: 60(\mathrm{v} / \mathrm{v}) \text { at a gradient program. Flow rate: } \\
0.25 \mathrm{~mL} / \mathrm{min} \text {. Rt: } 0.75 \mathrm{~min} \text {. }\end{array}$ & UV (220 nm) & Tablets and capsules & Uddin et al. (2015) \\
\hline HPLC & $\begin{array}{l}\text { Shim-pack VP-ODS column }(250 \times 4.6 \mathrm{~mm}) \text {. Mobile phase: } \\
\text { phosphate buffer } \mathrm{pH} 4.4 \text { and methanol }(91: 9, \mathrm{v} / \mathrm{v}) \text {. Flow rate: } \\
2.0 \mathrm{~mL} / \mathrm{min} . \mathrm{Rt}: 4.9 \mathrm{~min} \text {. }\end{array}$ & UV $(220 \mathrm{~nm})$ & Tablets & $\begin{array}{l}\text { Muchlisyam and Alfan } \\
\text { (2014) }\end{array}$ \\
\hline $\begin{array}{l}\text { Capillary } \\
\quad \text { electrophoresis }\end{array}$ & $\begin{array}{l}\text { Polyimidecoated fused silica-capillaries of } 56 \mathrm{~cm} \text { (effective length: } \\
48 \mathrm{~cm} \text { ) } \times 50 \mu \mathrm{m} \text { I.D. (Agilent). Electrolyte: } 25 \mathrm{mM} \text { sodium } \\
\text { tetraborate buffer and } 100 \mathrm{mM} \text { sodium dodecyl sulphate, pH } \\
9.3 \text {, as surfactant. Voltage: }+25 \mathrm{kV} \text {. Temperature: } 25^{\circ} \mathrm{C} \text {. } \\
\text { Migration time: } 3.41 \mathrm{~min} \text {. }\end{array}$ & UV $(210 \mathrm{~nm})$ & Raw material & Simon et al. (2014) \\
\hline lodometry & Potassium monopersulfate was used as reagent. & Not applicable & Raw material & Blazhevskii et al. (2013) \\
\hline HPLC & $\begin{array}{l}\mathrm{C}_{18} \text { ACE column }(150 \times 4.6 \mathrm{~mm} ; 3 \mu \mathrm{m}) \text {. Mobile phase: } \\
\text { phosphoric acid solution and acetonitrile }(90: 10 \mathrm{v} / \mathrm{v}) \text {. Flow } \\
\text { rate: } 1.0 \mathrm{~mL} / \mathrm{min} \text {. Rt: } 10.0 \mathrm{~min} \text {. }\end{array}$ & UV (220 nm) & Veterinary medicines & Miguel et al. (2013) \\
\hline HPLC & $\begin{array}{l}\mathrm{C}_{18} \text { Hypersil ODS column }(250 \times 4.6 \mathrm{~mm}) \text {. Mobile phase: acetate } \\
\text { buffer } \mathrm{pH} 4.0 \text { and methanol }(94: 6 \mathrm{v} / \mathrm{v}) \text {. Flow rate: } 1.0 \mathrm{~mL} / \mathrm{min} \text {. } \\
\text { Rt: } 7.03 \mathrm{~min} \text {. }\end{array}$ & UV $(228 \mathrm{~nm})$ & Injectable & Rajesh et al. (2013) \\
\hline HPLC & $\begin{array}{l}\text { MG Capacel Pak } \mathrm{C}_{18} \text { column. Mobile phase: phosphate buffer } \\
\text { solution and methanol }(50: 50, \mathrm{v} / \mathrm{v}), \mathrm{pH} 3.0 \text {. Flow rate: } 1.0 \mathrm{~mL} / \\
\text { min. }\end{array}$ & UV (229 nm) & $\begin{array}{c}\text { Bulk and pharmaceutical } \\
\text { dosage forms }\end{array}$ & Beg et al. (2012) \\
\hline HPLC & $\begin{array}{l}\text { Phenomenex Luna } \mathrm{C}_{18}(2) \text { column }(250 \times 4.6 \mathrm{~mm} ; 5 \mu \mathrm{m}) \text {. Mobile } \\
\text { Phase: } 25 \mathrm{mM} / \mathrm{L} \text { potassium dihydrogen phosphate, }(\mathrm{pH} \\
\text { adjusted to } 4.5 \text { with orthophosphoric acid) and acetonitrile } \\
\text { (40:60, } / \mathrm{v}) \text {. Flow rate: } 1.0 \mathrm{~mL} / \mathrm{min} \text {. Rt: } 2.053 \mathrm{~min} \text {. }\end{array}$ & UV $(238 \mathrm{~nm})$ & Tablets & Bojaraju et al. (2012) \\
\hline HPLC & $\begin{array}{l}\text { Dikma Diamonsil } \mathrm{C}_{18} \text { column }(250 \times 4.6 \mathrm{~mm} ; 10 \mu \mathrm{m}) \text {, at } 40^{\circ} \mathrm{C} \text {. } \\
\text { Mobile phase: phosphoric acid buffer solution }(\mathrm{pH} 5.0) \text { and } \\
\text { acetonitrile }(98: 2 \mathrm{v} / \mathrm{v})\end{array}$ & UV $(254 \mathrm{~nm})$ & Oral suspension & Lu et al. (2012) \\
\hline HPLC & $\begin{array}{l}\text { Symmetry XTERRA } C_{18} \text { column }(150 \times 4.6 \mathrm{~mm} ; 5 \mu \mathrm{m}) \text {. Mobile } \\
\text { phase: phosphate buffer solution }(\mathrm{pH} 2.5) \text { and acetonitrile } \\
(40: 60, \mathrm{v} / \mathrm{v}) \text {. Flow rate: } 0.8 \mathrm{~mL} / \mathrm{min} \text {. Rt: } 4.1 \mathrm{~min} \text {. }\end{array}$ & UV $(228 \mathrm{~nm})$ & Tablets & Nadiminti et al. (2012) \\
\hline HPLC & $\begin{array}{l}\text { Inertsil ODS- } 2 \text { column }(150 \times 4.6 \mathrm{~mm} ; 5 \mu \mathrm{m}) \text {, at } 25^{\circ} \mathrm{C} \text {. Mobile } \\
\text { phase: methanol and } 0.01 \mathrm{M} \text { ammonium acetate }(52: 48, \mathrm{v} / \mathrm{v}) \text {. } \\
\text { Flow rate: } 1.0 \mathrm{~mL} / \mathrm{min} \text {. }\end{array}$ & UV (247 nm) & Dry suspensions & Song and Chen (2012) \\
\hline HPLC & $\begin{array}{l}\text { Kromasil } \mathrm{C}_{18} \text { column. Mobile phase: } 0.05 \mathrm{M} \text { monosodium } \\
\text { phosphate and methanol }(95: 5, \mathrm{v} / \mathrm{v}) \text {. Flow rate: } 1.0 \mathrm{~mL} / \mathrm{min} \text {. }\end{array}$ & UV $(220 \mathrm{~nm})$ & Injectable & Zou et al. (2012) \\
\hline HPLC & $\begin{array}{l}\text { Capcell Pak } \mathrm{C}_{18} \text { column. Mobile phase: methanol and } 0.02 \mathrm{M} \\
\text { phosphate buffer solution (50:50, v/v, pH 3.5). Flow rate: } \\
1.0 \mathrm{~mL} / \mathrm{min} \text {. Rt: } 3.99 \mathrm{~min} \text {. }\end{array}$ & UV (229 nm) & Tablets & Beg et al., 2011 \\
\hline HPLC & Agilent Zorbax Eclipse Plus $C_{18}$ column $(150 \times 2.1 \mathrm{~mm} ; 3.5 \mu \mathrm{m})$ & MS & Raw material & Dinha et al. (2011) \\
\hline
\end{tabular}
at $35^{\circ} \mathrm{C}$. Mobile phase: solution $\mathrm{A}$ (water and $0.1 \%$ formic acid) and solution $B$ (acetonitrile and $0.1 \%$ formic acid) $(90: 10, v / v)$. Flow rate: $9.0-11.0 \mathrm{~mL} / \mathrm{min}$. 
Table 1. (Continued).

\begin{tabular}{|c|c|c|c|c|}
\hline Method & Conditions & Detection system & Matrix & Reference \\
\hline HPLC & $\begin{array}{l}\mathrm{C}_{18} \text { column. Mobile phase: solution } \mathrm{A}(0.02 \mathrm{M} \text { monopotassium } \\
\text { phosphate, pH adjusted to } 6.5 \text { with } 2 \mathrm{M} \mathrm{NaOH} \text { containing } \\
1.5 \% \text { cetyltrimethylammonium bromide, and acetonitrile } \\
[60: 40, \mathrm{v} / \mathrm{v}]) \text { and solution } \mathrm{B}(0.02 \mathrm{M} \text { monopotassium } \\
\text { phosphate, pH adjusted to } 6.5 \text { with the same solution of } \\
\mathrm{NaOH} \text { and acetonitrile }[30: 70, \mathrm{v} / \mathrm{v}])\end{array}$ & UV (254 nm) & Granules & Lu and Wang (2011) \\
\hline HPLC & $\begin{array}{l}\mathrm{C}_{18} \text { Hypersil column }(250 \times 4.6 \mathrm{~mm} ; 5 \mu \mathrm{m}) \text {. Mobile phase: } \\
\text { potassium phosphate monobasic and methanol }(95: 05, \mathrm{v} / \mathrm{v}) \text {. } \\
\text { Flow rate: } 1.0 \mathrm{~mL} / \mathrm{min} \text {. Rt: } 6.30 \mathrm{~min} \text {. }\end{array}$ & UV (283 nm) & Pharmaceutical preparation & Manzoor et al. (2011) \\
\hline HPLC & $\begin{array}{l}\mathrm{C}_{18} \text { column HiQ Sil }(250 \times 4.6 \mathrm{~mm} ; 5 \mu \mathrm{m}) \text {. Mobile phase: } \\
\text { methanol and } 0.02 \mathrm{M} \text { ammonium acetate }(90: 10, \mathrm{v} / \mathrm{v}) \text { with } \mathrm{pH} \\
5.0 \text { adjusted with } 10 \% \text { orthophosphoric acid. Flow rate: } \\
1.0 \mathrm{~mL} / \mathrm{min} \text {. }\end{array}$ & UV (254 nm) & Capsules & Dhoka et al. (2010) \\
\hline HPLC & $\begin{array}{l}\mathrm{C}_{18} \text { Apollo column }(150 \times 4.6 \mathrm{~mm} ; 5 \mu \mathrm{m}) \text { Mobile phase: } 0.01 \mathrm{M} \\
\text { potassium phosphate monobasic and methanol }(45: 55, \mathrm{v} / \mathrm{v}) \\
\text { Flow rate: } 1.0 \mathrm{~mL} / \mathrm{min} \text {. Rt: } 1.57 \mathrm{~min} .\end{array}$ & UV/DAD (225 nm) & Capsules & Giang and Honag (2010) \\
\hline HPLC & $\begin{array}{l}\mathrm{C}_{18} \text { Hypersil column }(250 \times 4.6 \mathrm{~mm} ; 5 \mu \mathrm{m}) \text {. Mobile phase: } \\
\text { phosphate buffer and methanol }(95: 05, \mathrm{v} / \mathrm{v}) \text {. Flow rate: } \\
1.0 \mathrm{~mL} / \mathrm{min} \text {. }\end{array}$ & UV (220 nm) & Capsules & Jayakar et al. (2010) \\
\hline HPLC & $\begin{array}{l}\text { VertiSep }^{\text {TM }} \text { GES ODS column }(150 \times 4.5 \mathrm{~mm} ; 5 \mu \mathrm{m}) \text {. Mobile } \\
\text { phase: monobasic potassium phosphate solution, pH } 5.0 \text { and } \\
\text { acetonitrile }(96: 4, \mathrm{v} / \mathrm{v}) \text {. Flow rate: } 0.8 \mathrm{~mL} / \mathrm{min} \text {. }\end{array}$ & UV/DAD (230 nm) & Raw material & Rojanarata et al. (2010) \\
\hline HPLC & $\begin{array}{l}\mathrm{C}_{18} \text { column }(250 \times 4.5 \mathrm{~mm} ; 5 \mu \mathrm{m}) \text {. Mobile phase: methanol and } \\
\text { glacial acetic acid }(50: 50, \mathrm{v} / \mathrm{v}) \text {. Flow rate: } 1.0 \mathrm{~mL} / \mathrm{min} \text {. Rt: } \\
3.04 \mathrm{~min} .\end{array}$ & UV (254 nm) & Oily suspension & Sonawane and Bari (2010) \\
\hline HPLC & $\begin{array}{l}\mathrm{C}_{18} \text { Inertsil column }(250 \times 4.0 \mathrm{~mm} ; 4 \mu \mathrm{m}) \text {. Mobile phase: } \\
\text { monopotassium phosphate with } \mathrm{pH} \text { adjusted to } 5.0 \text { with } \\
\text { orthophosphoric acid, and methanol }(95: 5, \mathrm{v} / \mathrm{v}) \text {. Flow rate: } \\
1.0 \mathrm{~mL} / \mathrm{min} \text {. Rt: } 7.5 \mathrm{~min} \text {. }\end{array}$ & UV (220 nm) & Injectable & Tippa and Singh (2010) \\
\hline HPLC & $\begin{array}{l}\text { Spherisorb ODS- } 2 \text { column }(250 \times 4 \mathrm{~mm} ; 5 \mu \mathrm{m}) \text { at } 40^{\circ} \mathrm{C} \text {. Mobile } \\
\text { phase: phosphate buffer with pH } 3.5 \text { and acetonitrile }(35: 65 \text {, } \\
\text { v/v). Rt: } 4.0 \text { min. }\end{array}$ & UV (355 nm) & Raw material & Delis et al. (2009) \\
\hline HPLC & $\begin{array}{l}\mathrm{C}_{18} \text { Kromasil column }(250 \times 4.6 \mathrm{~mm} ; 5 \mu \mathrm{m}) \text {. Mobile phase: } \\
0.02 \mathrm{M} \text { dihydrogen potassium orthophosphate and } \\
\text { acetonitrile }(75: 25, \mathrm{v} / \mathrm{v}) \text {. Flow rate: } 1.5 \mathrm{~mL} / \mathrm{min} \text {. }\end{array}$ & UV (225 nm) & Capsules & Nikam et al. (2009) \\
\hline HPLC & $\begin{array}{l}\text { Luna } \mathrm{C} 18 \text { column }(150 \times 4.6 \mathrm{~mm} ; 5 \mu \mathrm{m}) \text { at } 50^{\circ} \mathrm{C} \text {. Mobile phase: } \\
\text { acetonitrile and water }(25: 75, \mathrm{v} / \mathrm{v}) \text {. Flow rate: } 1.5 \mathrm{~mL} / \mathrm{min} \text {. Rt: } \\
5.0 \mathrm{~min} \text {. }\end{array}$ & UV $(220 \mathrm{~nm})$ & Standard & Peña et al. (2009) \\
\hline HPLC & $\begin{array}{l}\mathrm{C}_{18} \text { Kromasil column }(150 \times 4.6 \mathrm{~mm} ; 5 \mu \mathrm{m}) \text {. Mobile phase: } \\
\text { acetonitrile and } 0.02 \mathrm{M} \text { potassium phosphate monobasic. } \\
\text { Flow rate: } 1.0 \mathrm{~mL} / \mathrm{min} \text {. }\end{array}$ & UV (230 nm) & Capsules & Qi et al. (2009) \\
\hline HPLC & $\begin{array}{l}\mathrm{C}_{18} \text { YMG column }(250 \times 4.6 \mathrm{~mm} ; 10 \mu \mathrm{m}) \text {. Mobile phase: } 0.015 \mathrm{M} \\
\text { sodium dodecyl sulfate and } 0.03 \mathrm{M} \text { sodium acetate }(\mathrm{pH} \\
\text { adjusted to } 4.2 \text { with acetic acid) and methanol }(80: 20, \mathrm{v} / \mathrm{v}) \text {. } \\
\text { Flow rate: } 1.0 \mathrm{~mL} / \mathrm{min} \text {. }\end{array}$ & UV (225 nm) & Capsules & Qin et al. (2009) \\
\hline HPLC & $\begin{array}{l}\mathrm{C}_{18} \text { column. Mobile phase: } 0.05 \mathrm{M} \text { potassium phosphate } \\
\text { monobasic and acetonitrile }(97.5: 2.5, \mathrm{v} / \mathrm{v}) \text {. Flow rate: } 1.0 \mathrm{~mL} / \\
\text { min. }\end{array}$ & UV (230 nm) & Granules & Hong et al. (2009) \\
\hline HPLC & $\begin{array}{l}\text { Shim-Pack VP-ODS column }(150 \times 4.6 \mathrm{~mm} ; 5 \mu \mathrm{m}) \text {. Mobile phase: } \\
\text { phosphate buffer pH } 5.0 \text { and ethanol }(90: 10, \mathrm{v} / \mathrm{v}) \text {. Flow rate: } \\
1.0 \mathrm{~mL} / \mathrm{min} .\end{array}$ & UV (228 nm) & Capsules & Li et al. (2008) \\
\hline DRIFTS & $\begin{array}{l}\text { The analysis was conducted in the mid-infrared region. Data were } \\
\text { analyzed using regression by partial least squares. }\end{array}$ & Not applicable & $\begin{array}{l}\text { Mixture of amoxicillin and } \\
\text { starch }\end{array}$ & Parisotto et al. (2007) \\
\hline HPLC & $\begin{array}{l}\text { Whatman Par tisil } 5 \text { ODS-3 column }(100 \times 4.6 \mathrm{~mm} ; 5 \mu \mathrm{m}) \text {. Mobile } \\
\text { phase: } 0.05 \mathrm{M} \text { phosphate buffer } \mathrm{pH} 4.0 \text { and methanol }(95: 5, \mathrm{v} / \\
\text { v). Flow rate: } 1.5 \mathrm{~mL} / \mathrm{min} \text {. Rt: } 3.2 \mathrm{~min} \text {. }\end{array}$ & UV (254 nm) & Tablets & Tavakoli et al. (2007) \\
\hline HPLC & $\begin{array}{l}\mathrm{C}_{18} \text { Phenomenex column }(150 \times 4.6 \mathrm{~mm} ; 5 \mu \mathrm{m}) \text { at } 30^{\circ} \mathrm{C} \text {. Mobile } \\
\text { phase: } 0.78 \% \text { monosodium phosphate } \mathrm{pH} 4.4 \text { and methanol } \\
(95: 5, \mathrm{v} / \mathrm{v}) \text {. Flow rate: } 0.8 \mathrm{~mL} / \mathrm{min} \text {. }\end{array}$ & UV (220 nm) & Granules & Zhang (2007) \\
\hline HPLC & $\begin{array}{l}\text { Shimadzu VP-ODS column }(250 \times 4.6 \mathrm{~mm}) \text {. Mobile phase: } \\
0.008 \mathrm{M} \text { sodium dodecyl sulfate }(\mathrm{pH} \text { adjusted to } 4.5 \text { with } \\
\text { phosphoric acid) and methanol }(68: 32, \mathrm{v} / \mathrm{v}) \text {. Flow rate: } 1.0 \mathrm{~mL} / \\
\text { min. }\end{array}$ & UV (225 nm) & Capsules & Hong et al. (2006) \\
\hline HPLC & $\begin{array}{l}\text { Gel filtration Ohpak SB-802.5 HQ column. Mobile phase: } \\
\text { phosphate buffer solution. Flow rate: } 0.8 \mathrm{~mL} / \mathrm{min} \text {. }\end{array}$ & Not available & Capsules and suspensions & Li et al. (2006) \\
\hline HPLC & $\begin{array}{l}\mathrm{C}_{18} \text { Nucleosil } 120 \text { column }(250 \times 4.6 \mathrm{~mm} ; 10 \mu \mathrm{m}) \text {. Mobile phase: } \\
\text { solution } \mathrm{A} \text { (methanol) and solution } \mathrm{B} \text { (buffer solution } \mathrm{pH} 3.0) \text {. }\end{array}$ & UV/DAD (230 nm) & $\begin{array}{l}\text { Veterinary formulation } \\
\left.\qquad \text { (Premix }^{\mathrm{TM}}\right)\end{array}$ & Pérez-Lozano et al. (2006) \\
\hline
\end{tabular}


Table 1. (Continued).

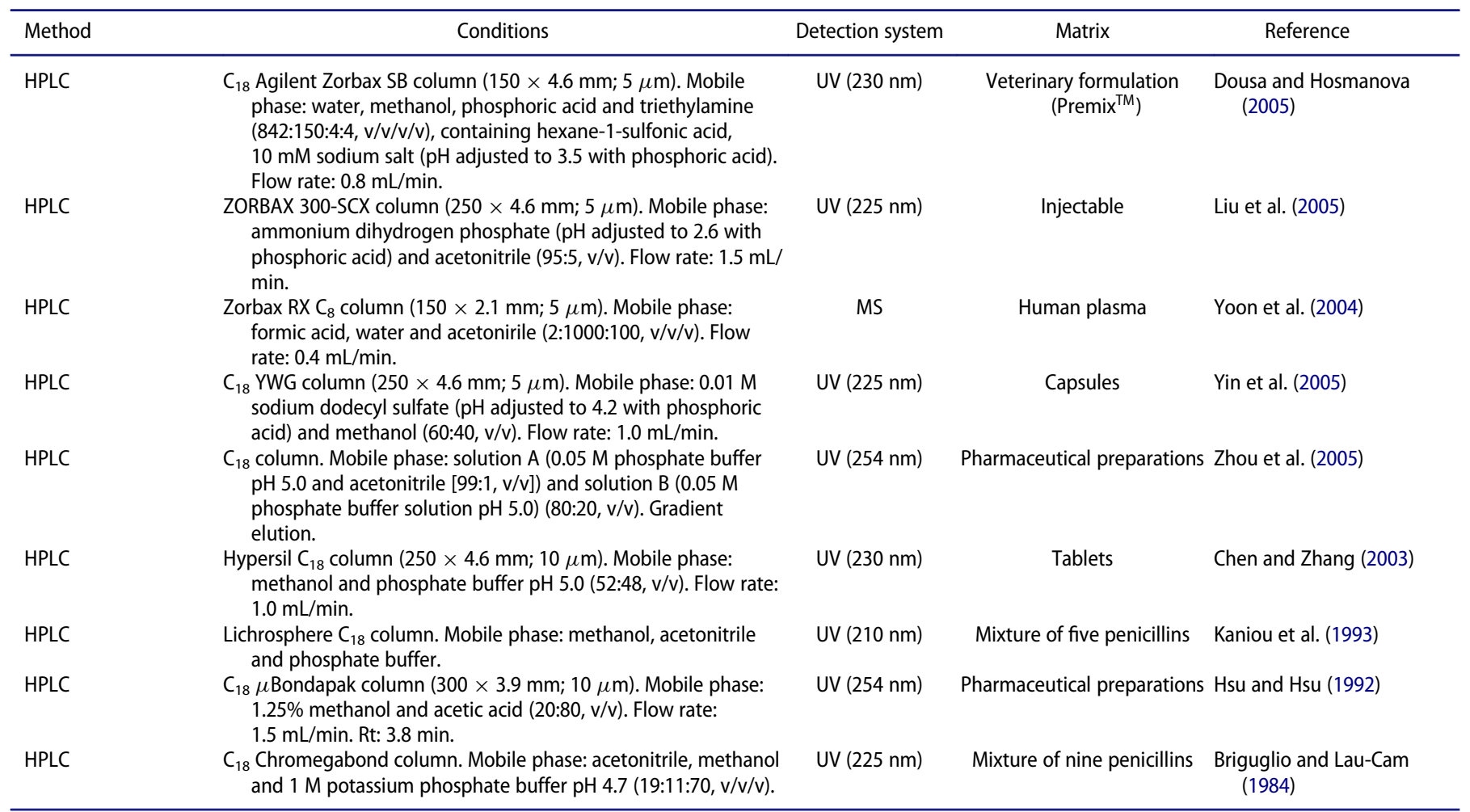

PIXE: Particle Induced X-ray Emission; HPLC: High performance liquid chromatography; U-HPLC: Ultra-high performance liquid chromatography; UV: ultraviolet detector; DAD: diode-array detector; DRIFTS: Diffuse reflectance infrared Fourier transform; MS: Mass spectrometry.

When orally administered, it keeps stable in acid medium; it is well absorbed by gastrointestinal tract and shows good penetration into tissues, such as the liver, lungs, prostate gland, muscle and synovial, pleural and amniotic fluids, being able to cross the placenta. Inflammations generally cause an increase of meningeal permeability to penicillins. Amoxicilin has a halflife of elimination of approximately 1.0-1.5 hours, and the kidney is its main route of elimination (Katzung, 2010; Kaur et al., 2011; Brazil, 2016b).

Concomitant use of probenecid and amoxicillin causes delays in the excretion of the penicillin. About $10-25 \%$ of the drug is eliminated as inactivated penicilloic acid, while the remainder is excreted in its unchanged form (Kaur et al., 2011).

\section{Physicochemical properties}

Amoxicillin is a semi-synthetic antibiotic belonging to the class of penicillins and subclass of aminopenicillins. Its hydrate form is used for the pharmaceutical dosage form of capsule. The hydrate form is chemically known as (2S,5R,6R)-6-[(2R)-2-amino-2-(4-hydroxyphenyl)-acetylamino]-3,3-dimethyl-7-oxo-4-thia-1-azabicyclo[3.2.0] heptane2-carboxylic acid trihydrate (The Merck Index, 2006; USP 37, 2014). Its molecular formula is $\mathrm{C}_{16} \mathrm{H}_{19} \mathrm{~N}_{3} \mathrm{O}_{5} \mathrm{~S}$, with molecular mass of $365.404 \mathrm{~g} / \mathrm{mol}$ (Brazilian Pharmacopoeia, 2010).

Amoxicillin has poor solubility in water, ethanol and methanol; it is insoluble in benzene, ethyl acetate, chloroform, diethyl ether and acetonitrile, and presents high solubility in hydroxide alkali solution. Its melting point is $194^{\circ} \mathrm{C}$ and its dissociation constants (pKa) are 2.4 (carboxylic acid), 7.4 (amine) and 9.6 (phenol) (Brazilian Pharmacopoeia, 2010; Rolinson and Geddes, 2007; The Merck Index, 2006; USP 37, 2014).

\section{Analytical methods for the analysis of amoxicillin}

The development of analytical methods for the analysis of amoxicillin in different matrixes is very relevant, mainly to assist bioavailability, bioequivalence and pharmacokinetic studies, as well as monitoring the quality of the marketed product.

There are several studies described in literature regarding the development of analytical methods for qualitative and quantitative analysis of amoxicillin using high-performance liquid chromatography (HPLC) (in most cases), ultra-performance liquid chromatography (U-HPLC), iodometry, diffuse reflectance infrared Fourier Transform spectroscopy (DRIFTS), capillary electrophoresis, particle induced X-ray emission (PIXE), colorimetry, among others. These methods aim to analyze amoxicillin in different matrixes, such as pharmaceutical dosage forms, raw material, biological fluids, food of animal origin, among others.

Table 1 presents the analytical methods found in literature for the analysis of amoxicillin in pharmaceutical dosage forms and raw material. Table 2 shows the analytical methods found to determine amoxicillin in biological fluids, food of animal origin, and other matrixes. Table 3 presents the methods suggested by pharmacopoeial compendia.

Regarding the analytical methods described in literature for the analysis of amoxicillin in different matrixes, it is 
Table 2. Analytical methods described in literature for determination of amoxicillin in biological fluids, food of animal origin and other matrixes.

\begin{tabular}{|c|c|c|c|c|}
\hline Method & Conditions & Detection system & Matrix & Reference \\
\hline U-HPLC & $\begin{array}{l}\text { Acquity UPLC }{ }^{\mathrm{TM}} \text { BEH RP } \mathrm{C}_{18} \text { analytical column }(50 \times 2.1 \mathrm{~mm} \text {; } \\
1.7 \mu \mathrm{m}) \text {. Mobile phase: solvent } \mathrm{A}(0.1 \% \text { formic acid in water }) \\
\text { and solvent } \mathrm{B}(0.1 \% \text { formic acid in acetonitrile), under a } \\
\text { gradient program. Flow rate: } 0.3 \mathrm{~mL} / \mathrm{min} \text {. Rt: } 1.87 \mathrm{~min} \text {. }\end{array}$ & MS & Cows' milk & Liu et al. (2016) \\
\hline HPLC & $\begin{array}{l}\text { Agilent } C_{18} \text { analytical column }(50 \times 4.6 \mathrm{~mm} ; 5 \mu \mathrm{m}) \text { protected } \\
\text { with a guard column }\left(C_{18} ; \text { particle size } 5 \mu \mathrm{m}\right) \text {. Mobile phase: } \\
\text { solvent } A \text { (acetonitrile) and solvent } B(0.1 \% \text { formic acid in } \\
\text { water), under a gradient program. Flow rate: } 1.0 \mathrm{~mL} / \mathrm{min} \text {. Rt: } \\
4.99 \mathrm{~min} \text {. }\end{array}$ & MS & Eggs & Sun et al. (2016) \\
\hline HPLC & $\begin{array}{l}\mathrm{C}_{18} \text { endcapped column }(70 \times 4.6 \mathrm{~mm} ; 5 \mu \mathrm{m}) \text {, coupled to a } \mathrm{C}_{18} \\
\text { pre-column }(4.0 \times 4.6 \mathrm{~mm}) \text {, at } 25^{\circ} \mathrm{C} \text {. Mobile phase: solution } \mathrm{A} \\
(50 \mathrm{mM} \text { phosphate buffer } \mathrm{pH} 3.0) \text { and solution } \mathrm{B} \text { (methanol). } \\
\text { Flow rate: } 1.0 \mathrm{~mL} / \mathrm{min} \text { and gradient elution. Rt: } 1.69 \mathrm{~min} \text {. }\end{array}$ & $\begin{array}{l}\text { UV/DAD } \\
(230 \mathrm{~nm})\end{array}$ & Residual water & Vosough et al. (2015) \\
\hline HPLC & $\begin{array}{l}\text { Thermo Hypersil } \mathrm{BDS}_{-} \mathrm{C}_{8} \text { analytical column }(250 \times 4.6 \mathrm{~mm} \text {; } \\
5 \mu \mathrm{m}) \text {. Mobile phase: phosphate buffer } \mathrm{pH} 7.0 \text { and } \\
\text { acetonitrile }(70: 30, \mathrm{v} / \mathrm{v}) \text {. Flow rate: } 1.0 \mathrm{~mL} / \mathrm{min} \text {. Rt: } 2.36 \mathrm{~min} \text {. }\end{array}$ & $\begin{array}{l}\text { UV/DAD } \\
(230 \mathrm{~nm})\end{array}$ & Simulated intestinal fluid & Sabry et al. (2015) \\
\hline U-HPLC & $\begin{array}{l}\text { Acquity UPLC BEH } C_{18} \text { column }(100 \times 2.1 \mathrm{~mm}) \text {. Mobile phase: } \\
\text { metanol and } 0.1 \% \text { formic acid solution. Flow rate: } 0.3 \mathrm{~mL} / \mathrm{min} \text {. } \\
\text { Rt: } 6.0 \mathrm{~min} \text {. }\end{array}$ & MS & $\begin{array}{l}\text { Human urine, serum, } \\
\text { cerebrospinal fluid and } \\
\text { bronchial aspiration }\end{array}$ & Carzola et al. (2014) \\
\hline HPLC & $\begin{array}{l}\mathrm{C}_{18} \text { ODS-BP column }(250 \times 4.6 \mathrm{~mm} ; 5 \mu \mathrm{m}) \text { coupled to a } \mathrm{C}_{18} \text { pre- } \\
\text { column, at } 30^{\circ} \mathrm{C} \text {. Mobile phase: } 0.1 \mathrm{M} \text { acetonitrile solution and } \\
\text { phosphate buffer solution } \mathrm{pH} 6.5(25: 75, \mathrm{v} / \mathrm{v}) \text {, containing } \\
0.015 \mathrm{M} \text { tetrabutylammonium bromide and sodium } \\
\text { thiosulfate. Flow rate: } 1.0 \mathrm{~mL} / \mathrm{min} \text {. }\end{array}$ & UV (325 nm) & Eggs & Ma et al. (2014) \\
\hline HPLC & $\begin{array}{l}\text { Acquity } \mathrm{HSS} \mathrm{T}_{3} \text { column }(50 \times 2.1 \mathrm{~mm} ; 1.7 \mu \mathrm{m}) \text {. Mobile phase: } \\
\text { solution A (acetic acid and amonium acetate buffer) and } \\
\text { solution B (acetonitrile). Flow rate: } 0.6 \mathrm{~mL} / \mathrm{min} \text {. Rt: } 5.0 \mathrm{~min} \text {. }\end{array}$ & MS & Human plasma & Collin et al. (2013) \\
\hline HPLC & $\begin{array}{l}\text { HyPURITY advance } \mathrm{C}_{18} \text { column }(50 \times 4.6 \mathrm{~mm} ; 5 \mu \mathrm{m}) \text {. Mobile } \\
\text { phase: ammonium acetate and acetonitrile }(20: 80, \mathrm{v} / \mathrm{v}) \text {. Flow } \\
\text { rate: } 1.5 \mathrm{~mL} / \mathrm{min} \text {. }\end{array}$ & MS & Human plasma & Gaikwad et al. (2013) \\
\hline HPLC & $\begin{array}{l}\mathrm{C}_{18} \mathrm{ACE} \text { column }(150 \times 4.6 \mathrm{~mm} ; 3 \mu \mathrm{m}) \text {. Mobile phase: } \\
\text { acetonitrile and water. Flow rate: } 0.4 \mathrm{~mL} / \mathrm{min} \text {. Rt: } 9 \mathrm{~min} \text {. }\end{array}$ & MS & Human blood & Szultka et al. (2013a) \\
\hline HPLC & $\begin{array}{l}\mathrm{C}_{8} \text { ACE column }(150 \times 4.6 \mathrm{~mm} ; 5 \mu \mathrm{m}) \text {. Mobile phase: methanol } \\
\text { and water }(50: 50, \mathrm{v} / \mathrm{v}) \text {. Flow rate: } 0.45 \mathrm{~mL} / \mathrm{min} \text {. Rt: } 7 \mathrm{~min} \text {. }\end{array}$ & MS & Human plasma & Szultka et al. (2013b) \\
\hline HPLC & $\begin{array}{l}\mathrm{C}_{18} \text { Athena column }(250 \times 4.6 \mathrm{~mm} ; 5 \mu \mathrm{m}) \text {. Mobile phase: } \\
\text { solution } \mathrm{A} \text { (acetonitrile) and solution } \mathrm{B}(0.01 \mathrm{M} \text { dihydrogen } \\
\text { phosphate). Flow rate: } 1.0 \mathrm{~mL} / \mathrm{min} .\end{array}$ & Fluorimetry & Albumine & Xie et al. (2013) \\
\hline U-HPLC & $\begin{array}{l}\mathrm{C}_{18} \text { column }(100 \times 2.1 \mathrm{~mm} ; 1.7 \mu \mathrm{m}) \text {. Mobile phase: formic acid in } \\
\text { water and formic acid in acetonitrile. Gradient elution. Flow } \\
\text { rate: } 0.4 \mathrm{~mL} / \mathrm{min} . \mathrm{Rt}: 5 \mathrm{~min} \text {. }\end{array}$ & MS & Human plasma & Carlier et al. (2012) \\
\hline U-HPLC & $\begin{array}{l}\text { Acquity UPLC BEH Waters } \mathrm{C}_{18} \text { column }(50 \times 2.1 \mathrm{~mm} ; 1.7 \mu \mathrm{m}) \text { at } \\
30^{\circ} \mathrm{C} \text {. Mobile phase: } 0.1 \% \text { formic acid in water and } 0.1 \% \\
\text { formic acid in acetonitrile }(50: 50, \mathrm{v} / \mathrm{v}) \text {. Flow rate: } 0.3 \mathrm{~mL} / \mathrm{min} \text {. }\end{array}$ & MS & Bovine milk & Li et al. (2012) \\
\hline HPLC & $\begin{array}{l}\text { Mobile phase: } 0.01 \mathrm{M} \text { monopotassium phosphate and } \\
\text { acetonitrile. Flow rate: } 1.0 \mathrm{~mL} / \mathrm{min} \text {. }\end{array}$ & Fluorimetry & Eggs & Xie et al. (2012) \\
\hline HPLC & $\begin{array}{l}\mathrm{C}_{18} \text { column. Mobile phase: solution A (methanol) and solution B } \\
(0.1 \% \text { formic acid). Gradient elution. }\end{array}$ & MS & Honey & Xu et al. (2012) \\
\hline HPLC & $\begin{array}{l}\mathrm{C}_{18} \text { HyPURITY column }(150 \times 2.1 \mathrm{~mm} ; 3.5 \mu \mathrm{m}) \text {. Mobile phase: } \\
0.1 \% \text { formic acid and acetonitrile. }\end{array}$ & MS & Fish derivatives & Guo et al. (2011) \\
\hline HPLC & $\begin{array}{l}\text { Waters Acquity UPLC HSS T } 3 \text { column }(100 \times 2.1 \mathrm{~mm} ; 1.8 \mu \mathrm{m}) \text { at } \\
30^{\circ} \mathrm{C} \text {. Mobile phase: acetonitrile, } 0.15 \% \text { formic acid in water } \\
\text { with } 5 \mathrm{mM} \text { ammonium acetate. Flow rate: } 0.25 \mathrm{~mL} / \mathrm{min} \text {. }\end{array}$ & MS & Bovine milk & Liu et al. (2011) \\
\hline HPLC & $\begin{array}{l}\text { Waters Atlantis } \mathrm{T}_{3} \text { column }(150 \times 2.1 \mathrm{~mm} ; 3 \mu \mathrm{m}) \text {. Mobile phase: } \\
\text { solution } \mathrm{A} \text { (acetonitrile) and solution } \mathrm{B} \text { (water with } 0.005 \% \\
\text { formic acid). Gradient elution. Flow rate: } 0.2 \mathrm{~mL} / \mathrm{min} \text {. Rt: } \\
8.0 \mathrm{~min} \text {. }\end{array}$ & MS & Bovine muscle & Lugoboni et al. (2011) \\
\hline HPLC & $\begin{array}{l}\text { Inertsil ODS column }(150 \times 4.6 \mathrm{~mm} ; 5 \mu \mathrm{m}) \text { at } 30^{\circ} \mathrm{C} \text {. Mobile } \\
\text { phase: mixture of } 30 \mathrm{mM} \text { potassium dihydrogen phosphate } \\
\text { buffer }(\mathrm{pH} 2.8) \text { and acetonitrile }(97.5: 2.5, \mathrm{v} / \mathrm{v}) \text {. Flow rate: } \\
1.0 \mathrm{~mL} / \mathrm{min} \text {. Rt: } 6.0 \mathrm{~min} \text {. }\end{array}$ & $\begin{array}{l}\text { UV/DAD } \\
(210 \mathrm{~nm})\end{array}$ & Human plasma & Pei et al. (2011) \\
\hline HPLC & $\begin{array}{l}\text { Atlantis } \mathrm{T}_{3} \text { column }(150 \times 4.6 \mathrm{~mm} ; 5 \mu \mathrm{m}) \text { coupled to a pre- } \\
\text { column }(20 \times 4.6 \mathrm{~mm} ; 5 \mu \mathrm{m}) \text {. Mobile phase: solution } \mathrm{A} \\
\text { ( } 10 \mathrm{mM} \text { phosphoric acid solution, adjusted to } \mathrm{pH} 2.0 \text { with } \mathrm{HCl}) \\
\text { and solution } \mathrm{B} \text { (acetonitrile). Flow rate: } 2.0 \mathrm{~mL} / \mathrm{min} \text { and } \\
\text { gradient elution. Rt: } 3.4 \mathrm{~min} \text {. }\end{array}$ & UV (230 nm) & Human plasma & Verdier et al. (2011) \\
\hline HPLC & $\begin{array}{l}\mathrm{C}_{18} \text { column }(250 \mathrm{~mm} \times 4.6 \mathrm{~nm} ; 5 \mu \mathrm{m}) \text {. Mobile phase: potassium } \\
\text { phosphate monobasic buffer and acetonitrile }(96: 4 \mathrm{v} / \mathrm{v}) \text {. Flow } \\
\text { rate: } 1.5 \mathrm{~mL} / \mathrm{min} \text {. }\end{array}$ & UV (230 nm) & Cleaning waste & $\begin{array}{l}\text { Gomes and Souza } \\
\text { (2010) }\end{array}$ \\
\hline
\end{tabular}


Table 2. (Continued).

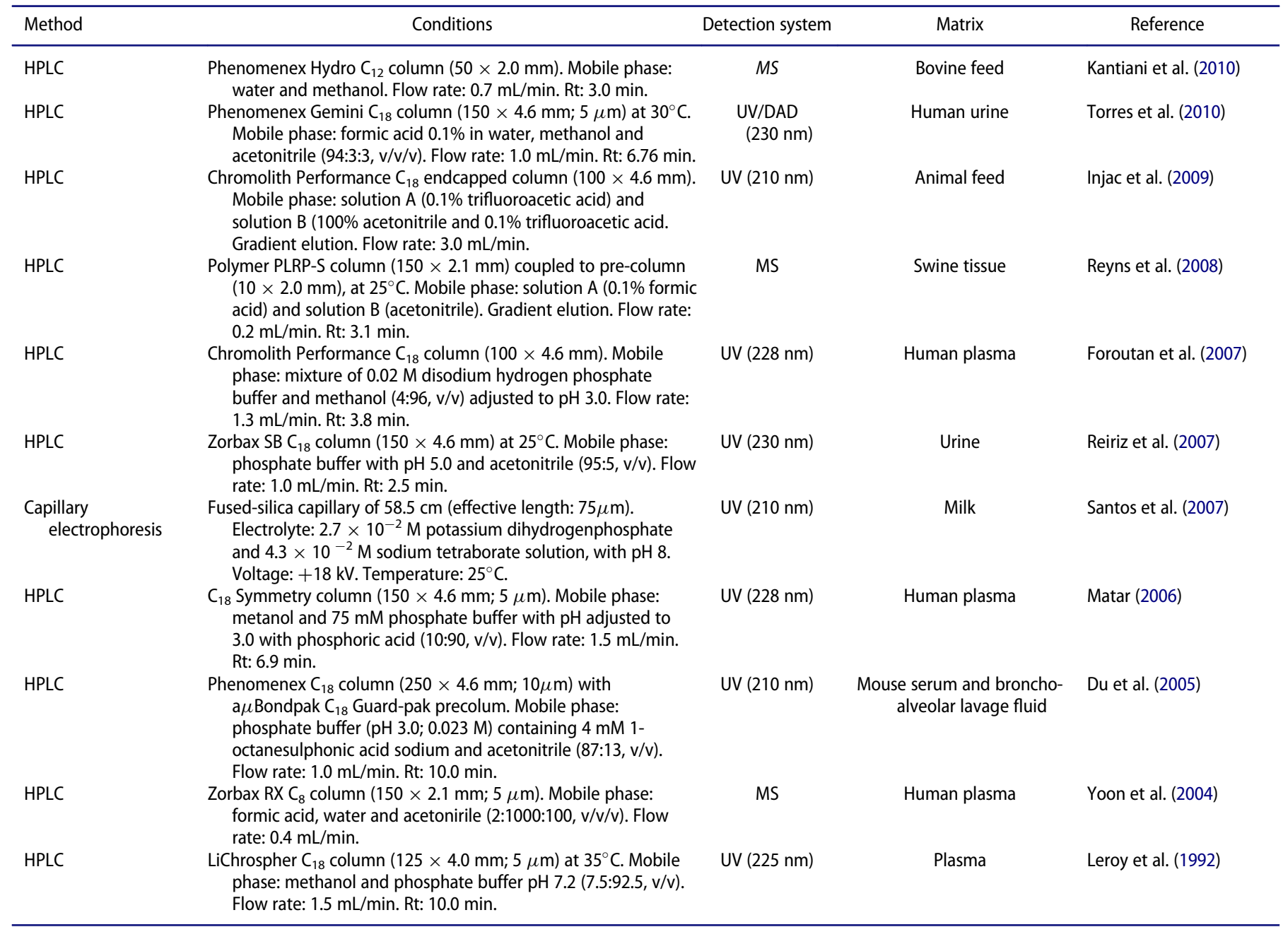

HPLC: High performance liquid chromatography; U-HPLC: Ultra-high performance liquid chromatography; UV: ultraviolet detector; DAD: diode-array detector; MS: Mass spectrometry

possible to observe that HPLC is the most used method. However, this technique has some disadvantages that need to be mentioned. It requires expensive equipment and analytical columns, makes use of large volumes of solvents as mobile phase, and it is a method of high maintenance cost. In addition, this method can be harmful to the environment, since most papers found in literature describe the use of toxic organic solvents, such as methanol and acetonitrile, as mobile phases. In these studies, the mobile phases were adjusted to $\mathrm{pH}$ between 2.5 and 6.5 , and the retention times ranged from 2.053 to 10.0 minutes. Some papers describe the use of $\mathrm{pH}$-correcting agents, such as formic acid, phosphoric acid and trifluoroacetic acid.

On the other hand, environmentally friendly analytical methods have been identified, such as capillary electrophoresis (Santos et al., 2007; Simon et al., 2014), spectroscopy by diffuse reflectance infrared Fourier transform (Parisotto et al., 2007) and U-HPLC (Li et al., 2012; Carlier et al., 2012; Carzola et al., 2014; Uddin et al., 2015; Liu et al., 2016). Furthermore, Li et al. (2008) describe a green HPLC method for determination of amoxicillin in capsules using ethanol as organic phase of the mobile phase, and this solvent is less harmful to operator's health and the environment. However, the suggested mobile phase has phosphate buffer solution ( $\mathrm{pH}$ 5.0). The use of buffer solution can damage the chromatographic system and the chromatographic column due to precipitation of salts. After using buffer solutions, the operators have to perform long washes in the chromatographic system in order to avoid such problems, which leads to greater waste of time, energy and water for cleaning (Santos-Neto, 2009, 2010; Tótoli and Salgado, 2014, 2015; Rodrigues and Salgado, 2016).

The detection system adopted by most HPLC methods was ultraviolet, in the wavelength ranged between 210 and $325 \mathrm{~nm}$. Fluorometry was also used, as well as diode array detector (DAD) and mass spectrometry. The used stationary phases are commonly $\mathrm{C}_{18}$ analytical columns.

The choice of reliable analytical methods for the analysis of medicines is extremely important. These methods must be continuously optimized, aiming to improve patient safety, reduce production costs and electric energy consumption, and minimize the impacts on the environment and health risks to operators. Green Chemistry is highly relevant nowadays, since it contributes directly to the environmental and economic impacts, sustainable social production and good management practices (Anastas, 2016; Hodges, 2016; Nies, 2016). 
Table 3. Analytical methods described in pharmacopoeial compendia for determination of amoxicillin.

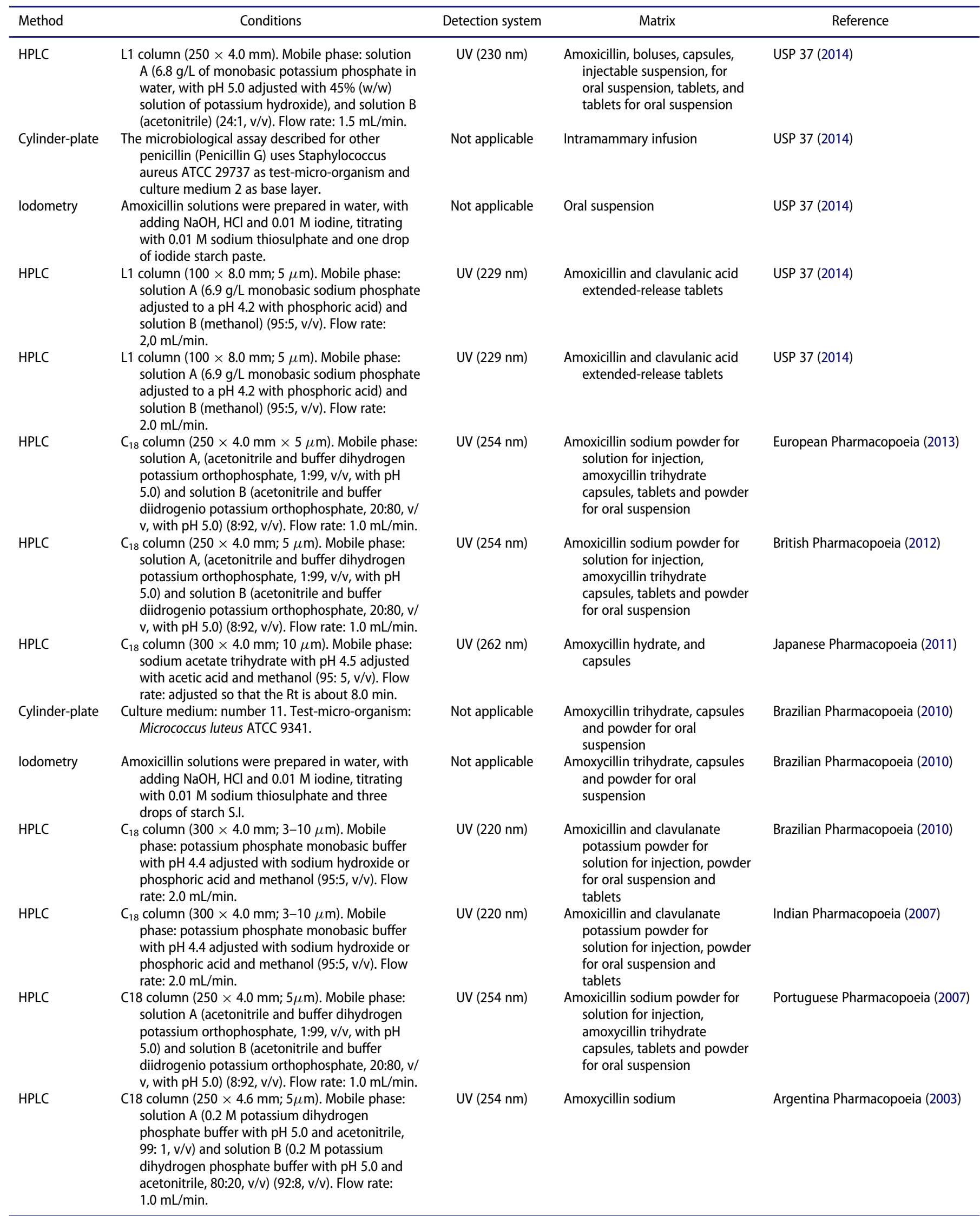

HPLC: High performance liquid chromatography. 


\section{Conclusion}

Amoxicillin belongs to the class of penicillins and the subclass of aminopenicillins, and it is a bactericidal antimicrobial agent with broad spectrum of action. Considering that it is widely used worldwide, the development and validation of reliable analytical methods for its identification and quantification is highly relevant. It is critical to maintaining the characteristics of the active pharmaceutical ingredient and patient's safety. In addition, antimicrobials with lack of quality contribute to the emergence of the serious problem of bacterial resistance.

Even though there are numerous analytical methods for the analysis of this penicillin, most of them use toxic organic solvents and techniques not focused on Green Chemistry. Thus, it is of utmost importance to insert a progressive vision for implementing new environmentally friendly methods for the quality control of this antimicrobial agent, which also contributes to social and economical aspects.

\section{Conflict of interest}

The authors declare no conflicts of interest.

\section{Funding}

This work was supported by PACD-FCFAr-UNESP (Araraquara, Brazil), CNPq (Brasília, Brazil), FAPESP (São Paulo, Brazil) and CAPES (Brasília, Brazil).

\section{ORCID}

Bianca Aparecida de Marco (ID http://orcid.org/0000-0003-2978-4892

\section{References}

Akhond, M.; Absalan, G.; Ershadifar, H. Highly Sensitive Colorimetric Determination of Amoxicillin in Pharmaceutical Formulations Based on Induced Aggregation of Gold Nanoparticles. Spectrochi. Acta Part A Mole. Biomol. Spectro. 2015, 143, 223-229.

Anastas, P. Redesigning the Material Basis of our Society and Our Economy. In Green \& Sustainable Chemistry Conference, Berlin, 2016

Argentina Pharmacopoeia. 7th ed. Buenos Aires Farmacopea Nacional Argentina 2003.

Beg, S.; Hasnain, M. S.; Swain, S.; Kohli, K. Validated Stability-Indicating LC Method for Estimation of Amoxicillin Trihydrate in Pharmaceutical Dosage Forms and Time-Dependent Release Formulations. Interna. J. Pharma. Sci. Nanotech. 2011, 4, 1423-1427.

Beg, S.; Kohli, K.; Swain, S.; Hasnain, M. S. Development and Validation of RP-HPLC Method for Quantitation of Amoxicillin Trihydrate in Bulk and Pharmaceutical Formulations Using Box-Behnken Experimental Design. J. Liq. Chroma. Rel. Tech. 2012, 35, 393-406.

Bejjani, A.; Roumié, M.; Akkad, S.; El-Yazbi, F.; Nsouli, B. Simultaneous Quantification of Amoxicillin and Potassium Clavulanate in Different Commercial Drugs Using PIXE Technique. Nuc. Instr. Methods Phy. Res. Sec. B Beam Interac. Mat. Atoms 2016, 371, 392-395.

BlazhevskiI, N. E.; Karpova, S. P.; KabachyI, V. I. Quantitative Determination of Penicillins by Iodometry Using Potassium Hydrogen Peroxymonosulfate. Antibio. Khimi. 2013, 58, 3-7.

Bojaraju, V.; Sireesha, D.; Prasad, V. V. L. N.; Diwan, P. V. Reverse Phase High Performance Liquid Chromatography Method for the Simultaneous Estimation of Amoxicillin Trihydrate and Tinidazole in the Tablet Dosage Form. Wor. J. Chem. 2012, 7, 47-52.

BP. British Pharmacopoeia; Her Majesty's Stationary Office: London, 2012. v.1.
Brazil. Brazilian Health Surveillance Agency (ANVISA). Antimicrobials main groups available for clinical use. Available at: http://www.anvisa. gov.br/servicosaude/controle/rede_rm/cursos/rm_controle/opas_web/ modulo1/penicilinas3.htm. Accessed April 13, 2016a.

Brazil. Brazilian Health Surveillance Agency (ANVISA). Electronic leaflet (Bulário virtual). Available at: www.anvisa.gov.br. Accessed June 13, $2016 b$.

Brazil. Brazilian Health Surveillance Agency (ANVISA). Reference medicines registered /List A. Available at: http://www.anvisa.gov.br/medica mentos/referencia/lmr_a.pdf. Accessed June 13, 2016c.

Brazil. Brazilian Health Surveillance Agency (ANVISA). Reference medicines registered /List B. Available at: http://www.anvisa.gov.br/medica mentos/referencia/lmr_b.pdf. Accessed May 4, 2016d.

Brazilian Pharmacopoeia. 5th ed. Brasília, ANVISA, 2010.

Briguglio, G. T.; Lau-Cam, C. A. Separation and Identification of Nine Penicillins by Reverse Phase Liquid Chromatography. J. Asso. Off. Analyt. Chemi. 1984, 67, 228-231.

Brunton, L. L.; Lazo, J. S.; Parker, K. L. Goodman \& Gilman: The Pharmacological Basis of Therapeutics; McGraw Hill: Rio de Janeiro, Brazil, 2012.

Carlier, M.; Fogão, V.; Roberts, J. A.; Van de Velde, E.; De Waele, J. J.; Verstraete, A. G. Quantification Seven $\beta$-Lactam Antibiotic and Two $\beta$-Lactamase Inhibitors in Human Plasma, A Method Using UPLCEM/EM Validated. Interna. J. Antimicro. Agents 2012, 40, 416-422.

Carzola, R. R.; Romero, G. R.; Frenich, A. G.; Rodrigues, M. A.; Martínez, J. L. The Simultaneous Analysis of Antibiotics in Biological Samples by Liquid Spectrometry of High Efficiency Ultra-Tandem Mass Chromatography. J. Pharma. Biomed. Analy. 2014, 89, 203-212.

Chen, H.; Zhang, D. RP-HPLC Determination of Amoxicillin and Dicloxacillin in Tablets. Yaowu Fenxi Zazhi 2003, 23, 51-53.

Collin, P.; De Bock, L.; T’Jollyn, H.; Boussery, K.; Van Bocxlaer, J. Development and Validation of a Fast and Uniform Approach to Quantify $\beta$-Lactam 55 Antibiotics in Human Plasma by Solid Phase ExtractionLiquid Chromatography-Electrospray-Tandem Mass Spectrometry. Talanta 2013, 103, 285-293.

Delis, G.; Batzias, G.; Kounenis, G.; Papadopoulou, M. K. Application and Validation of a LC/Fluorescence Method for the Determination of Amoxicillin in Sheep Serum and Tissue Cage Fluid. J. Pharm. Biomed. Analy. 2009, 49, 375-380.

Dinha, Q. T.; Alliot, F.; Guigona, E. M.; Eurina, J.; Chevreuila, M.; Labadieb, P. Measurement of Trace Levels of Antibiotics in River Water Using On-Line Enrichment and Triple-Quadrupole LC-MS/ MS. Talanta 2011, 85, 1238-1245.

Dhoka, M.; Gawande, V.; Joshi, P. High Performance Liquid Chromatographic Method for Determination of Amoxicillin Trihydrate and Bromhexine Hydrochloride in Oral Dosage Forms. Interna. J. Pharm. Pharm. Sci. 2010, 2, 129-133.

Dousa, M.; Hosmanova, R. Rapid Determination of Amoxicillin in Premixes by HPLC. J. Pharm. Biomed. Analy. 2005, 37, 373-377.

Du, X.; Li, C.; Sun, H. K.; Nightingale, C. H.; Nicolau, D. P. A Sensitive Assay of Amoxicillin in Mouse Serum and Broncho-Alveolar Lavage Fluid by Liquid-Liquid Extraction and Reversed-Phase HPLC. J. Pharm. Biomed. Analy. 2005, 39, 648-652.

EP. European Pharmacopoeia; 8th ed. Strasbourg, 2013. v.2.

Foroutan, S. M.; Zarghi, A.; Shafaati, A.; Khoddam, A.; Movahed, H. Simultaneous Determination of Amoxicillin and Clavulanic Acid in Human Plasma by Isocratic Reversed-Phase HPLC Using UV Detection. J. Pharm. Biomed. Analy. 2007, 45, 531-534.

Gaikwad, A.; Gavali, G.; Narendiran, A. S.; Katale, D.; Bonde, S.; Bhadane, R. P. An LC-MS-MS Method for the Simultaneous Quantification of Amoxicillin and Clavulanic Acid in Human Plasma and its Pharmacokinetic Application. J. Pharm. Res. 2013, 6, 804-812.

Giang, D. T.; Hoang, V. D. Comparative Study of RP-HPLC and UV Spectrophotometric Techniques for the Simultaneous Determination of Amoxicillin and Cloxacillin in Capsules. J. Young Pharm. 2010, 2, 190195.

Gomes, M. L. P. C.; Souza, S. V. C. Method Validation for the Determination of Amoxicillin Residues Applied to Cleaning Validation in the Pharmaceutical Industry Penicillanic. New Chem. 2010, 33(4), 972977. 
Guo, M.; Li, Z.; Tan, Z.; Wu, H.; Han, X.; Leng, K.; Zhai, Y. Determination of Residues of 8 Penicillins in Fishery Products by Dispersive SolidPhase Extraction and Liquid Chromatography-Tandem Mass Spectrometry. Fenxi Ceshi Xuebao 2011, 30, 969-975.

Hodges, P. Green Chemistry \& the Economy of the Future. In Green \& Sustainable Chemistry Conference; Berlin, 2016.

Hoefel, H. H. K.; Lautert, L. Intravenous Administration of Antibiotics and Bacterial Resistance: Nursing Responsibility. Nurs. Electr. J. 2006, 8, 441-449.

Holten, K. B.; Onsusko, E. M. Appropriate Prescribing of Oral $\beta$-Lactam Antibiotics. Am. Fam. Physi. 2000, 63, 1557-1565.

Hong, J.; Wei, X.; Yu, C. Analysis of Amoxicillin and Dicloxacillin by HPLC. Guangdong Yaoxueyuan Xuebao 2006, 22, 624-626.

Hong, J.; Li, Q.; Wang, Y. Quality Control of Amoxicillin Granules by HPLC. Guangdong Yaoxueyuan Xuebao 2009, 25, 42-45.

Hsu, M. C.; Hsu, R. W. High Performance Liquid Chromatographic Method for Potency Determination of Amoxicillin in Commercial Preparations and for Stability Studies. Antimicro. Agents Chemother. 1992, 36, 1276-1279.

Injac, R.; Kocevar, N.; Strukelj, B. Optimized Method for Determination of Amoxicillin, Ampicillin, Sulfamethoxazole, and Sulfacetamide in Animal Feed by Micellar Electrokinetic Capillary Chromatography and Comparison with High-Performance Liquid Chromatography. Croatica Chemica Acta 2009, 82, 685-694.

IP. Indian Pharmacopoeia; 5th ed. Indian Pharmacopoeia Commission: New Delhi, 2007.

Jayakar, B.; Kumudhavalli, M. V.; Chandira, R. M.; Kumar, M; Saravanan, C. Method Development and Validation of RP-HPLC Method for Simultaneous Determination of Amoxicillin and Potassium Clavulanate. Internat. J. PharmTech Res. 2010, 2, 906-909.

JP. Japanese Pharmacopoeia; 16th ed. Society of Japanese Pharmacopoeia: Tokyo, 2011.

Kaniou, I. P.; Zachariadis, G. A.; Stratis, J. A. Separation and Determination of Five Penicillins by Reversed-Phase HPLC. J. Liq. Chromato. 1993, 16, 2891-2897.

Kantiani, L.; Farré, M.; Grases, J. M. F.; Barceló, D. Development and Validation of a Pressurised Liquid Extraction Liquid Chromatography-ElectrosprayTandem Mass Spectrometry Method for Beta-Lactams and Sulfonamides in Animal Feed. J. Chromato. A 2010, 1217, 4247-4254.

Katzung, B. G. Basic and Clinical Pharmacology; 10th ed. AMGH: Porto Alegre, 2010.

Kaur, S. P.; Rao, R.; Nanda, S. Amoxicillin: A Broad Spectrum Antibiotic. Interna. J. Pharm. Pharm. Sci. 2011, 3, 30-37.

Konari, S. N.; Jacob, J. T. Stability-Indicating LC-Analytical Method Development and Validation for the Simultaneous Estimation of Flucloxacillin and Amoxicillin in Pharmaceutical Dosage Form. J. Taibah Univ. Sci. 2015, 9, 167-176.

La Roca, M. F.; Soares Sobrinho, J. L.; Nunes, L. C. C.; Rolim Neto, P. J. Desenvolvimento e Validação de Método Analítico: Passo Importante na Produção de Medicamentos. Revista Brasileira de Farmácia 2007, $88,177-180$.

Leroy, P.; Gavriloff, C.; Nicolas, A.; Arhimbault, P; Ambroggi, G. Comparative Assay of Amoxicillin by High-Performance Liquid Chromatography and Microbiological Methods for Pharmacokinetic Studies in Calves. Interna. J. Pharm. 1992, 82, 157-164.

Li, H.; Xia, X.; Xue, Y.; Tang, S.; Xião, X.; Li, J.; Shen, J. Simultaneous Determination of Amoxicillin and Prednisolone in Bovine Milk Using Ultra-High Performance Liquid Chromatography Tandem Mass Spectrometry. J. Chromato. B 2012, 900, 59-63.

Li, Z. Y.; Li, Y. F.; Fan, B.; Xiang, J. L. Separation and Determination of Macromolecular Impurities in Amoxicillin. Zhongguo Xiandai Yingyong Yaoxue 2006, 23, 326-237.

Li, Y. W.; Gou, H. L.; Yang, X. H.; Wang, Z. Z. RP-HPLC Determination of Amoxicillin. Lihua Jianyan, Huaxue Fence 2008, 44, 216-217.

Liu, C.; Wangb, H.; Jiang, Y.; Du, Z. Rapid and Simultaneous Determination of Amoxicillin, Penicillin G, and their Major Metabolites in Bovine Milk by Ultra-High-Performance Liquid Chromatography-Tandem Mass Spectrometry. J. Chromato. B 2011, 879, 533-540.

Liu, H.; Wang, H.; Sunderland, V. B. An Isocratic Ion Exchange HPLC Method for the Simultaneous Determination of Flucloxacillin and
Amoxicillin in a Pharmaceutical Formulation for Injection. J. Pharm. Biomed. Analy. 2005, 37, 395-398.

Liu, Y.; Zhu, K.; Wang, J.; Huang, X.; Wang, G.; Li, C.; Cao, J.; Ding, S. Simultaneous Detection and Comparative Pharmacokinetics of Amoxicillin, Clavulanic Acid and Prednisolone in Cows' Milk by UPLC-MS/ MS. J. Chromato. B 2016, 1008, 74-80.

Lu, Q.; Wang, X. Determination of Flucloxacillin Magnesium and Amoxicillin Granules by HPLC. Zhongguo Yiyao Gongye Zazhi 2011, 42, 295-297.

Lu, L. Y.; Dai, L. P.; Zhong, P.; Zhang, Z. F. Determination of Related Substances in Flucloxacillin Magnesium and Amoxicillin Oral Suspension by HPLC. Xinan Minzu Daxue Xuebao 2012, 38, 92-96.

Lugoboni, B.; Gazzoti, T.; Zironi, E.; Barbarossa, A.; Pagliuca, G. Development and Validation of a Liquid Chromatography/Tandem Mass Spectrometry Method for Quantitative Determination of Amoxicillin in Bovine Muscle. J. Chromato. B 2011, 879, 1980-1986.

Ma, S.; Shi, Y.; Lv, H.; Shang, X. Development and Validation of a HPLC with Solid-Phase Clean-Up and Pre-Column Derivatization Method for the Simultaneous Determination of Three $\beta$-Lactam Antibiotics Residues in Eggs. Wor. J. Pharma. Res. 2014, 3, 33-44.

Manzoor, A.; Suresh, B. G.; Sathish, K. S. A. Development and Validation of Amoxicillin by RP-HPLC Method in Bulk Drug and Pharmaceutical Dosage Forms. Interna. J. ChemTech Res. 2011, 3, 1037-1041.

Matar, K. M. Simple and Rapid LC Method for the Determination of Amoxicillin in Plasma. Chromatographia 2006, 64, 255-260.

Miguel, G. C.; Orlando, E. A.; Simionato, A. V. C. Quantification of BetaLactam Antibiotics in Veterinary Drugs: Amoxicillin and Ampicillin Determination by High Performance Liquid Chromatography. New Chem. 2013, 36(8), 1214-1221.

Miller, E. L. The Penicillins: A Review and Update. J. Midwif. Women's Health 2002, 47, 426-434.

Muchlisyam, F. R. H.; Alfan, M. Optimization of Phosphate Buffer pH 4.4methanol as Mobile Phase for Analysis of Amoxicillin and Clavulanate Potassium Mixture in Tablets by High Performance Liquid Chromatography (HPLC). Interna. J. PharmTech Res. 2014, 6, 174-184.

Nadiminti, A.; Gunda, A.; Tupally, K. R.; Abbaraju, P. L.; Jakka, K.; Nagubandi, A. S. Simultaneous Estimation of Amoxicillin Trihydrate and Carbocysteine Drug Present in Formulation by RP-HPLC Method and its Validation. J. Pharm. Res. 2012, 5, 1889-1895.

Nies, A. Ethics, Legislation and Economics. In Green \& Sustainable Chemistry Conference; Berlin, 2016.

Nikam, D. S.; Bonde, C. G.; Surana, S. J.; Venkateshwarlu, G.; Dekate, P. G. Development and Validation of RP-HPLC Method for Simultaneous Estimation of Amoxicillin Trihydrate and Flucloxacillin Sodium in Capsule Dosage Form. Internat. J. PharmTech Res. 2009, 1, 935-939.

Parisotto, G.; Ferrão, M. F.; Furtado, J. C.; Molz, R. F. Determination of Amoxicillin Content in Powdered Pharmaceutical Formulations Using DRIFTS and PLS. J. Pharma. Sci. 2007, 43(1), 89-96.

Pei, Q.; Yang, G. -P.; Li, Z. -J.; Peng, X. -D.; Fan, J. -H.; Liu, Z. -Q. Simultaneous Analysis of Amoxicillin and Sulbactam in Human Plasma by HPLC-DAD for Assessment of Bioequivalence. J. Chromato. B 2011, 879, 2000-2004.

Peña, E. B.; Urraca, J. L.; Bondi, M. C. M. Quantitative Determination of Penicillin V and Amoxicillin in Feed Samples by Pressurised Liquid Extraction and Liquid Chromatography with Ultravioleta Detection. J. Pharm. Biomed. Analy. 2009, 49, 289-294.

Pérez-Lozano, P.; García-Montoy, A. E.; Orriols, A.; Miñarro, M.; Ticó, J. R.; Suñé-Negre, J. M. Stability Evaluation of Amoxicillin in a Solid Premix Veterinary Formulation by Monitoring the Degradation Products Through a New HPLC Analytical Method. J. Pharma. Biomed. Analy. 2006, 42, 192-199.

Petri, W. A. J. Antimicrobianos (continuação): Penicilinas, Cefalosporinase Outros Antibióticos $\beta$-Lactâmicos. In Goodman \& Gilman: Pharmacological Basis of Therapeutics; Hardman, J.G.; Limbird, L.E.; Gilman, A. G., Eds.; 11 th ed. McGraw-Hill: New York, 2006, pp. 891-910.

Portuguese Pharmacopoeia. 8th ed. Lisboa: Infarmed 2007.

Qi, Y.; Yang, Q.; Tong, Y.; Hao, L.; Wu, S. Determination of Compound Amoxicillin and Cloxacillin Sodium Capsules and Its Related Substances by HPLC. Zhongguo Yaoshi 2009, 12, 1174-1177.

Qin, F.; Liu, H.; Pan, Y. Improved Method for Determination of Amoxicillin and Dicloxacillin in Compound Capsules. Yaowu Fenxi Zazhi 2009, $29,121-124$. 
Rajesh, K. S.; Yadav, A. B.; Patel, A. Development and Validation of Stability Indicating HPLC Method for Estimation of Co-Amoxyclav Injection. Pharmagene 2013, 1, 60-64.

Reiriz, A. G.; Damiani, P. C.; Olivieri, A. C. Different Strategies for the Direct Determination of Amoxicillin in Human Urine by SecondOrder Multivariate Analysis of Kinetic-Spectrophotometric Data. Talanta 2007, 71, 806-815.

Reyns, T.; Cherlet, M.; De Baere, S.; De Backer, P.; Croubels, S. Rapid Method for the Quantification of Amoxicillin and Its Major Metabolites in Pig Tissues by Liquid Chromatography-Tandem Mass Spectrometry With Emphasis on Stability Issues. J.Chromato. B 2008, 861, 108-116.

Rodrigues, D. F.; Salgado, H. R. N. Development and Validation of a Green Analytical Method of RP-HPLC for Quantification Cefepime Hydrochloride in Pharmaceutical Dosage Forms: Simple, Sensitive and Economic. Curr. Pharm. Analy. 2016, 12, 306-314.

Rojanarata, T.; Opanasopit, P.; Ngawhirunpat, T.; Saehuan, C.; Wiyakrutta, S.; Meevootisomc, V. A Simple, Sensitive and Green Bienzymatic UVSpectrophotometric Assay of Amoxicillin Formulations. Enzy. Microb. Tech. 2010, 46, 292-296.

Rolinson, G. N.; Geddes, A. M. The 50th Anniversary of the Discovery of 6-Aminopenicillanic Acid (6-APA). Interna. J. Antimicro. Agents 2007, 29, 3-8.

Sabry, S. M.; Abdel-Hay, M. H.; Belal, T. S.; Mahgoub, A. A. Development and Validation of HPLC-DAD Method for the Simultaneous Determination of Amoxicillin, Metronidazole and Rabeprazole Sodium. Application to Spiked Simulated Intestinal Fluid Samples. Fr. Annal. Pharma. 2015, 73(5), 351-360.

Santos-Neto, A. J. Uma Visão Técnica Para a Compreensão e Resolução de Problemas em Sistemas de Cromatografia Líquida. Scientia Chromato. 2009, 1, 83-96.

Santos-Neto, A. J. Problemas com o Formato dos Picos em Cromatografia Líquida - Parte 4. Scientia Chromato. 2010, 2, 61-67.

Santos, S. M.; Henriques, M.; Duarte, A. C.; Esteves, V. I. Development and Application of a Capillary Electrophoresis Based Method for the Simultaneous Screening of Six Antibiotics in Spiked Milk Samples. Talanta 2007, 71, 731-737.

Simon, B.; Hancu, G.; Gyéresi, Á. Application of Capillary Electrophoresis to the Simultaneous Determination and Stability Study of Four Extensively Used Penicillin Derivatives. Br. J. Pharma. Sci. 2014, 50(3), 521-527.

Sonawane, L. V.; Bari, S. B. Development and Validation of RP-HPLC Method for the Simultaneous Estimation of Amoxicillin Trihydrate and Bromhexine Hydrochloride From Oily Suspension. Pharma. Analy. Acta 2010, 1, 107.

Song, J.; Chen, X. Preparation and Determination of Compound Amoxicillin Dry Suspensions. Zhongguo Yaoshi 2012, 15, 1606-1609.

Sun, L.; Jia, L.; Xie, X.; Xie, K.; Wang, J.; Liu, J.; Cui, L.; Zhang, G.; Dai, G.; Wang, J. Quantitative Analysis of Amoxicillin, Its Major Metabolites and Ampicillin in Eggs by Liquid Chromatography Combined With Electrospray Ionization Tandem Mass Spectrometry. Food Chem. 2016, 192, 313-318.

Szultka, M.; Krzeminski, R.; Szeliga, J.; Jackowski, M.; Buszewski, B. A New Approach for Antibiotic Drugs Determination in Human Plasma by Liquid Chromatography-Mass Spectrometry. J. Chromato. A 2013a, 1272, 41-49.

Szultka, M.; Krzeminski, R.; Szeliga, J.; Jackowski, M.; Buszewski, B. Simultaneous Determination of Selected Chemotherapeutics in Human Whole Blood by Molecularly Imprinted Polymers Coated Solid Phase Microextraction Fibers and Liquid Chromatography-Tandem Mass Spectrometry. J. Chromato. B 2013b, 940, 66-76.

Tavakoli, N.; Varshosaz, J.; Dorkoosh, F.; Zargarzadeh, M. R. Development and Validation of a Simple HPLC Method for Simultaneous in vitro Determination of Amoxicillin and Metronidazole at Single Wavelength. J. Pharma. Biomed. Analy. 2007, 43, 325-329.

The Merck Index: an encyclopedia of chemicals, drugs, and biological; 14th ed. Whitehouse Station: Merck \& Co., 2006.

Tippa, D. M. R.; Singh, N. Development and Validation of Stability Indicating HPLC Method for Simultaneous Estimation of Amoxicillin and Clavulanic Acid in Injection. Am. J. Analy. Chem. 2010, 1, 95-101.

Torres, R. F.; Consentino, M. O.; Lopez, M. A. B.; Mochon, M. C. Simultaneous Determination of 11 Antibiotics and Their Main Metabolites
From Four Different Groups by Reversed-Phase High-Performance Liquid Chromatography-Diode Array-Fluorescence (HPLC-DADFLD) in Human Urine Samples. Talanta 2010, 81, 871-880.

Tótoli, E. G.; Salgado, H. R. N. Development and Validation of an Economic, Environmental Friendly and Stability-Indicating Analytical Method for Determination of Ampicillin Sodium for Injection by RPHPLC. Wor. J. Phar. Pharma. Sci. 2014, 3(6), 1928-1943.

Tótoli, E. G.; Salgado, H. R. N. Development, Optimization, and Validation of a Green and Stability-Indicating HPLC Method for Determination of Daptomycin in Lyophilized Powder. J. AOAC Interna. 2015, 98, 1276-1285.

Uddin, M. N.; Das, S.; Khan, S. H.; Shill, S. K.; Bhuiyan, H. R.; Karim, R. Simultaneous Determination of Amoxicillin and Chloramphenicol and their Drug Interaction Study by the Validated UPLC Method. J. Taibah Univ. Sci. 2015, doi:10.1016/j.jtusci.2015.11.005.

USP 37. The United States Pharmacopeia. 37th ed. Rockville: The United States Pharmacopeial Convention, 2014.

Vaidya, H.; Patel, S.; Patel, D.; Pradhan, P. K.; Upadhyay, U. Analytical Method Development and Validation of Diloxanide Furoate and Ornidazole in its Combined Pharmaceutical Dosage Form. Schol. Acad. J. Pharmacy (SAJP) 2015, 4(9), 398-404.

Verdier, M. C.; Tribut, O.; Tattevin, P.; Tulzo, Y. L.; Michelet, C.; BentueFerrer, D. Simultaneous Determination of $12 \beta$-Lactam Antibiotics in Human Plasma by High-Performance Liquid Chromatography With UV Detection: Application to Therapeutic Drug Monitoring. Antimicro. Agents Chemo. 2011, 55, 4873-4879.

Vispute, A. C.; Patil, V. A.; Patil, D. A. RP-HPLC Method for Simultaneous Estimation of Amoxicillin and Carbocisteine in Dosage Forms. Pharmacia Lettre 2013, 5, 327-333.

Vosough, M.; Rashvand, M.; Esfahani, H. M.; Kargosha, K.; Salemi, A. Direct Analysis of Six Antibiotics in Waste Water Samples using Rapid High-Performance Liquidch Romatography Coupled with Diode Array Detector: A Chemometric Study Towards Green Analytical Chemistry. Talanta 2015, 135, 7-17.

Williams, D. A.; Lemke, T. I. Foye's Principles of Medicinal Chemistry; Wolters Kluwer Health/ Lippincott Williams \& Wilkins: Philadelphia, United States of America, 2013.

Xie, K. -Z.; Chen, X. -S.; Xu, D.; Guo, H. -S.; Jia, L. -F.; Xie, X.; Huang, Y. -P.; Zhang, X. -J.; Sun, Y. -Y.; Dai, G. -J.; Zhang, G. -X.; Wang, J. -Y. Determination of Amoxicillin Residues in Eggs by HPLC with Fluorescence Detection. Shipin Kexue 2012, 33, 264-268.

Xie, K.; Zhao, M.; Guo, H.; Zhang, X.; Sun, Y.; Li, A.; Xie, X.; Zhang, G.; Dai, G.; Wang, J. Determination and Depletion of Amoxicillin Residues in Eggs. Food Addit. Contam. 2013, 30(4), 670-677.

Xu, W.; Zhang, X.; Wu, B.; Yin, Y.; Yang, W.; Shen, C.; Ding, T.; Chen, H. Determination of Amoxicillin in Honey by High Performance Liquid Chromatography-Tandem Mass Spectrometry. Chin. J. Chromato. 2012, 30, 1089-1092.

Xue, Z. -H.; Liao, J. -H. Simultaneous Content Determination of Amoxicillin and Cloxacillin for Injection by HPLC. Zhongguo Kangshengsu Zazhi 2013, 38, 749-751.

Yin, L.; Le, J.; Hong, Z.; Hu, C. HPLC Determination of Amoxicillin and Dicloxacillin in Compound Capsules. Yaowu Fenxi Zazhi 2005, 25, 1395-1398.

Yoon, K. H.; Lee, S. Y.; Kim, W.; Park, J. S.; Kim, H. J. Simultaneous Determination of Amoxicillin and Clavulanic Acid in Human Plasma by HPLC-ESI Mass Spectrometry. J. Chromato. B 2004, 813, 121-127.

Zhang, K. Determination of Amoxicillin and Clavulanate Potassium Granules by HPLC. Yaoxue Jinzhan 2007, 31, 221-224.

Zhang, Y. -Z.; Fan, L. -L.; Qu, X. -S.; Feng, J.; Wei, Y. -B. Determination of Amoxicillin and Clavulanate Potassium Suspension (14:1) by RPHPLC. Anhui Yiyao 2013, 17, 207-210.

Zhou, X.; Gu, L.; Pang, H.; Hu, C.; Feng, F. Comparative Studies on Determination of Related Substances of Amoxicillin-Potassium Clavulanate and its Preparations. Zhongguo Kangshengsu Zazhi 2005, 30, 737-740.

Zou, C. -Y.; Wang, F. -Q.; Meng, X. -J. Determination of Amoxicillin Sodium and Clavulanate Potassium Content for Injection by HPLC. Liaoning Huagong 2012, 41, 426-428. 